\title{
Hábitos alimentares da toninha (Pontoporia blainvillei) na costa norte do Estado do Rio de Janeiro, Brasil
}

Esta nota de pesquisa descreve as presas identificadas no conteúdo estomacal de quatro carcaças de toninha, Pontoporia blainvillei, recuperadas a partir de encalhes em praias da costa norte do Rio de Janeiro, sudeste do Brasil, entre 2010 e 2011. Os peixes identificados como presas foram Lycengraulis grossidens, Trichiurus lepturus, Paralonchurus brasiliensis, Chirocentrodon bleekerianus, Isopisthus parvipinnis, Pellona harroweri e Stellifer rastrifer. Os cefalópodes consumidos foram as lulas Doryteuthis plei e D. sanpaulensis. Camarões da família Penaeidae cuja identificação específica não foi possível foram registrados nos conteúdos estomacais. Todas as presas são espécies costeiras, abundantes na região. A comparação com dados pretéritos sobre a alimentação de P. blainvillei, obtidos entre 1989 e 1999, sugere que não houve mudança temporal no seu hábito alimentar preferencial na região.

Palavras-chave: Pontoporia blainvillei; Toninha; Alimentação; Oceano Atlântico.

\section{Eating habits of porpoise (Pontoporia blainvillei) on the north coast of the State of Rio de Janeiro, Brazil}

This research note describes the prey identified in the stomach contents of four carcasses of Franciscana dolphin, Pontoporia blainvillei, recovered from strandings on beaches on the northern coast of Rio de Janeiro, southeastern Brazil, between 2010 and 2011. The fish identified as prey were Lycengraulis grossidens, Trichiurus lepturus, Paralonchurus brasiliensis, Chirocentrodon bleekerianus, Isopisthus parvipinnis, Pellona harroweri and Stellifer rastrifer. The cephalopods consumed were the squids Doryteuthis plei and D. sanpaulensis. Shrimps of the Penaeidae family whose specific identification was not possible were recorded in the stomach contents. All prey are coastal species, abundant in the region. The comparison with past data on the P. blainvillei diet, obtained between 1989 and 1999 , suggests that there was no temporal change in its preferred feeding habits in the region.

Keywords: Pontoporia blainvillei; Porpoise; Food; Atlantic Ocean.

Topic: Notas Científicas

Reviewed anonymously in the process of blind peer.
Received: 02/01/2021

Approved: 28/01/2021
Ana Paula Madeira Di Beneditto (iD)

Universidade Estadual do Norte Fluminense Darcy Ribeiro, Brasi http://lattes.cnpq.br/8402221071942314

http://orcid.org/0000-0002-4248-9380

anapaula@uenf.br

Salvatore Siciliano (iD)

Fundação Oswaldo Cruz, Brasil

http://lattes.cnpq.br/2471615656999141

http://orcid.org/0000-0002-0124-8070

gemmlagos@gmail.com
Referencing this:

BENEDITTO, A. P. M.; SICILIANO, S.. Hábitos alimentares da toninha (Pontoporia blainvillei) na costa norte do Estado do Rio de Janeiro, Brasil. Revista Ibero Americana de Ciências Ambientais, v.12, n.1, p.745-749, 2021. DOI: http://doi.org/10.6008/CBPC21796858.2021.001.0060

DOI: 10.6008/CBPC2179-6858.2021.001.0060 


\section{INTRODUÇÃO}

O golfinho Pontoporia blainvillei (Gervais \& d'Orbigny, 1844), conhecido popularmente como toninha ou franciscana, é o pequeno cetáceo mais vulnerável ao longo do Oceano Atlântico Sul-Ocidental devido a capturas incidentais em pescarias praticadas com redes de emalhe (OTT et al., 2002). O estado de conservação da espécie é 'vulnerável', de acordo com a classificação da União Internacional para a Conservação da Natureza - IUCN, com tendência populacional decrescente (ZERBINI et al., 2017). Portanto, ações que visam monitorar suas populações, incluindo estudos sobre história de vida e biologia são recomendadas. A toninha habita águas costeiras rasas, em geral até 30-50 m de profundidade, entre o sudeste do Brasil $\left(18^{\circ} 25^{\prime} \mathrm{S}\right)$ e a Argentina $\left(42^{\circ} 10^{\prime} \mathrm{S}\right)$, com preferência por regiões próximas a desembocaduras de rios e estuários (SICILIANO et al., 2002). Os autores verificaram que a espécie não se distribui continuamente ao longo desse intervalo latitudinal, com hiatos entre o centro e o sul do Rio de Janeiro, abrangendo quase $300 \mathrm{~km}$ de costa, e no sul do Espírito Santo, compreendendo aproximadamente $150 \mathrm{~km}$ de costa.

Estudos de longo prazo sobre o hábito alimentar de $P$. blainvillei indicaram que a alimentação é preferencialmente constituída de peixes ósseos e cefalópodes (lulas) costeiros, com até $10,0 \mathrm{~cm}$ de comprimento (BENEDITTO et al., 2001; DANILEWICZ et al., 2002; CAMPOS et al., 2020). No norte do Rio de Janeiro, sudeste do Brasil, Beneditto et al. (2001) identificaram 25 espécies de presas em 85 conteúdos estomacais analisados entre 1989 e 1999, caracterizando a alimentação de $P$. blainvillei na região. Os conteúdos estomacais foram provenientes de indivíduos mortos incidentalmente em pescarias. Dentre o total de presas consumidas, cinco se destacaram como preferenciais, com organismos associados ao fundo e a superfície, revelando alimentação do golfinho ao longo da coluna d'água. A análise do conteúdo estomacal de vertebrados mortos é um método tradicionalmente utilizado para identificação e quantificação da dieta. Na verdade, este é o método mais eficiente para o reconhecimento taxonômico e estimativas de tamanho de presas ingeridas, especialmente para vertebrados que se alimentam embaixo d"água, tais como golfinhos (BENEDITTO et al., 2011).

Esse estudo analisa o conteúdo estomacal de $P$. blainvillei a partir de carcaças encalhadas em praias da costa norte do Rio de Janeiro, sudeste do Brasil, entre 2010 e 2011. O objetivo é fornecer informações complementares sobre o hábito alimentar da espécie na região e verificar potencial mudança temporal em suas preferências alimentares.

\section{RELATO}

Levantamentos regulares em praias para monitoramento de encalhes de cetáceos foram realizados ao longo da costa norte do Rio de Janeiro em 2010 e 2011, resultando no registro de quatro carcaças de $P$. blainvillei no estágio de decomposição 2 - carcaça fresca (GERACl et al., 2005) (Figura 1). Dados de comprimento total e sexo das carcaças não foram registrados. Após a necropsia, o estômago foi retirado da cavidade abdominal e seu conteúdo lavado em água corrente, sobre peneira de $500 \mu \mathrm{m}$, para recuperação 
de restos das presas consumidas. Os restos recuperados foram otólitos de peixes ósseos, osso supraoccipital (no caso do peixe Trichiurus lepturus), mandíbulas de cefalópodes e carapaça de crustáceos, preservados a seco ou em solução de álcool 70\% para identificação taxonômica, quantificação e estimativa do tamanho corporal original das presas, de acordo com o protocolo estabelecido em Beneditto et al. (2001). Após a análise do conteúdo estomacal, os restos das presas foram descartados e as carcaças dos golfinhos depositadas no acervo do Grupo de Estudos de Mamíferos Marinhos da Região dos Lagos (GEMM-Lagos).

Sete espécies de peixes ósseos, duas espécies de cefalópodes (lulas) e camarões da família Penaeidae foram registrados nos conteúdos estomacais (Tabela 1). O número de espécies de presas em cada conteúdo estomacal variou entre 2 e 7, e o número de indivíduos de cada espécie variou de 1 a 49 (Tabela 1). A estimativa do tamanho original das presas consumidas indicou que a maior parte dos peixes era menor que 10,0 cm, com exceção de $T$. lepturus, cuja anatomia corporal é mais alongada em comparação com as demais espécies.

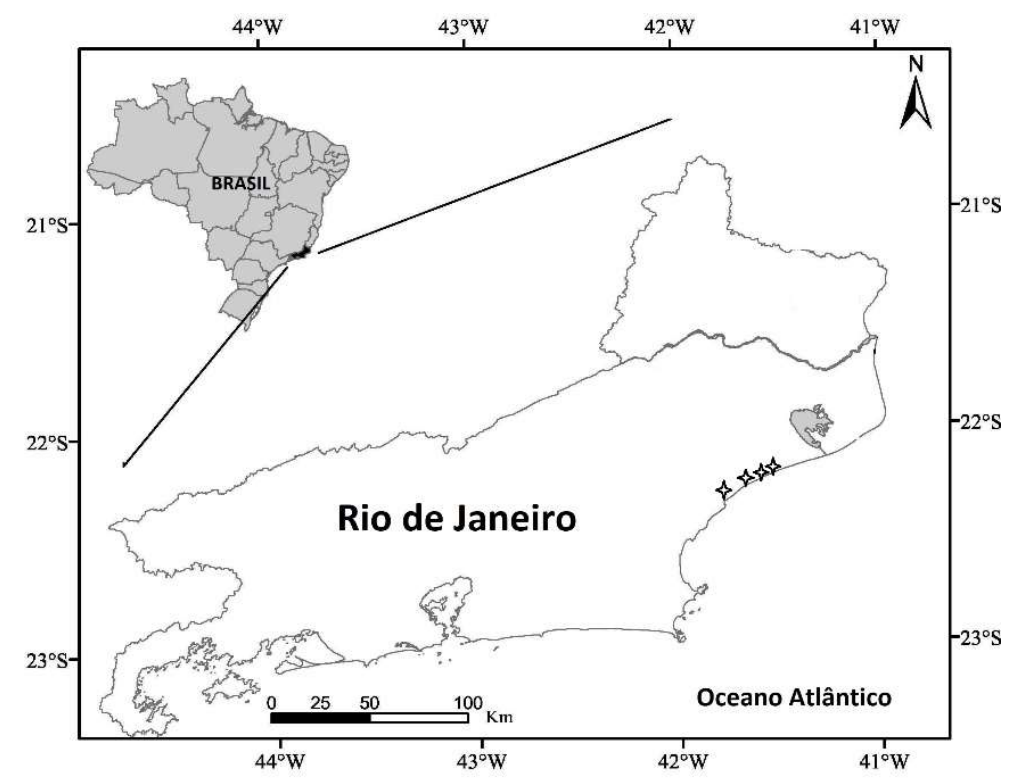

Figura 1: Mapa do Brasil e do estado do Rio de Janeiro, com destaque para os pontos de coleta das quatro carcaças de toninhas encalhadas (estrela).

Tabela 1: Presas identificadas em conteúdos estomacais de carcaças do golfinho Pontoporia blainvillei encalhadas em praias da costa norte do Rio de Janeiro, sudeste do Brasil, entre 2010 e 2011. O tamanho de T. lepturus é medido como comprimento total e das demais espécies de peixe como comprimento padrão. $O$ tamanho de $D$. plei e $D$. sanpaulensis é medido como comprimento do manto. DP: desvio padrão.

\begin{tabular}{|l|l|l|l|}
\hline Registro do golfinho e data de coleta & Espécies de presa & $\begin{array}{l}\text { Tamanho (cm) } \\
\text { Média } \pm \text { DP }\end{array}$ & Número de presas \\
\hline GEMM 240 & Lycengraulis grossidens (peixe) & $7,2 \pm 0,9$ & 31 \\
Novembro/2010 & Doryteuthis plei (lula) & 27,1 & 1 \\
\hline GEMM 241 & Lycengraulis grossidens (peixe) & $8,4 \pm 1,1$ & 5 \\
Novembro/2010 & Stellifer rastrifer (peixe) & $3,3 \pm 1,5$ & 8 \\
& Isopisthus parvipinnis (peixe) & $3,9 \pm 0,9$ & 49 \\
& Doryteuthis plei (lula) & 16,3 & 1 \\
& Penaeidae (camarão) & & 1 \\
\hline GEMM 256 & Trichiurus lepturus (peixe) & $45,5 \pm 8,9$ & 11 \\
Janeiro/2011 & Lycengraulis grossidens (peixe) & 9,7 & 1 \\
& Paralonchurus brasiliensis (peixe) & 8,9 & 1 \\
& Chirocentrodon bleekerianus (peixe) & 13,0 & 1 \\
\hline GEMM 271 & Doryteuthis plei (lula) & $20,2 \pm 4,0$ & 4 \\
\hline
\end{tabular}




\begin{tabular}{|l|l|l|l|}
\hline Fevereiro/2011 & Stellifer rastrifer (peixe) & $3,1 \pm 2,1$ & 3 \\
& Isopisthus parvipinnis (peixe) & $4,6 \pm 1,9$ & 6 \\
& Pellona harroweri (peixe) & 4,0 & 1 \\
& Doryteuthis plei (lula) & 10,2 & 1 \\
& D. sanpaulensis (lula) & $3,4 \pm 5,4$ & 10 \\
& Penaeidae (camarão) & & 15 \\
\hline
\end{tabular}

\section{DISCUSSÃO}

Todas as espécies de peixe e lulas identificadas nos conteúdos estomacais de $P$. blainvillei já foram registradas como presas do golfinho na região por Beneditto et al. (2001). Dentre os peixes consumidos, as autoras destacaram Stellifer sp., Anchoa filifera, P. harroweri and I. parvipinnis como as espécies mais importantes na dieta, e dentre os cefalópodes o destaque foi $D$. sanpaulensis. Em apenas quatro conteúdos estomacais analisados, o que representa $5 \%$ do que foi considerado no estudo anterior ( $n=85$ conteúdos estomacais), três dessas cinco espécies foram registradas, i.e. $60 \%$. Isso sugere que a preferência alimentar de $P$. blainvillei na região tem se mantido a mesma ao longo de mais de duas décadas, pelo menos (1989 até 2010-11). A manutenção dessa preferência é uma indicação indireta de que as populações de presas estão presentes em quantidades suficientes para sustentar seus predadores, nesse caso o golfinho $P$. blainvillei.

As presas de $P$. blainvillei na costa norte do Rio de Janeiro são espécies costeiras, abundantes na região durante todo ano, e a maioria é considerada descarte da pesca artesanal local voltada para a captura comercial de camarões (BENEDITTO et al., 1998; BENEDITTO et al., 2003). O consumo de presas associadas ao fundo, como peixes da família Sciaenidae (S. rastrifer, I. parvipinnis, P. brasiliensis), e presas pelágicas, tais como L. grossidens, C. bleekerianus e P. harroweri, por exemplo, confirmam o comportamento alimentar do golfinho ao longo da coluna d'água, já mencionado em Beneditto et al. (2001). Essa plasticidade para a captura das presas favorece a utilização dos recursos alimentares disponíveis no ambiente marinho costeiro, mas também deve compensar a limitação do golfinho quanto à ingestão de presas com tamanho corporal maior que $10,0 \mathrm{~cm}$.

$\mathrm{Na}$ área de estudo, P. blainvillei divide o ambiente costeiro e os recursos alimentares disponíveis com outros predadores, tais como o boto-cinza (Sotalia guianensis) e indivíduos adultos do peixe-espada, $T$. lepturus. No entanto, estudos prévios indicaram que esses organismos coexistem com baixo grau de sobreposição alimentar (BITTAR et al., 2009; BENEDITTO et al., 2011). Considerando que provavelmente a abundância de recursos alimentares na região não sofreu alteração ao longo do tempo e que a competição trófica com outros predadores é baixa, pode-se inferir que a principal ameaça à população de $P$. blainvillei que se distribui na costa norte do Rio de Janeiro continua sendo a captura incidental em pescarias com redes de emalhe praticadas sobre a plataforma continental, área de ocorrência preferencial da espécie.

\section{CONSIDERAÇÕES FINAIS}

Esta nota de pesquisa fornece informações complementares sobre o hábito alimentar do golfinho $P$. blainvillei, que é a espécie de pequeno cetáceo mais ameaçada do Oceano Atlântico Sul-Ocidental. Os dados de conteúdo estomacal, apesar de baseados em apenas quatro indivíduos, confirmam que a espécie é primariamente piscívora, mas que lulas também são presas importantes na sua dieta. A constância temporal 
das presas preferenciais indica que os recursos alimentares se mantêm disponíveis para a espécie na costa norte do Rio de Janeiro. Dessa forma, a disponibilidade de presas não é um fator que compromete a sua presença na região.

AGRADECIMENTOS: Ao Conselho Nacional de Desenvolvimento Científico e Tecnológico - CNPq (301.259/2017-8, 306.076/2019-5) e a Fundação Carlos Chagas Filho de Amparo à Pesquisa do Estado do Rio de Janeiro - FAPERJ (E-26/202.770/2017, E-26/210.064/2018, E-26/210.844/2018) pelo financiamento à pesquisa.

\section{REFERÊNCIAS}

BITTAR, V. T.; BENEDITTO, A. P. M.. Diet and potential feeding overlap between Trichiurus lepturus (Osteichthyes, Perciformes) and Pontoporia blainvillei (Mammalia, Cetacea) in northern Rio de Janeiro, Brazil. Zoologia, v.26, n.2, p.374378, 2009. DOI: https://doi.org/10.1590/S198446702009000200023

BENEDITTO, A. P. M.; RAMOS, R. M. A.. Biology and conservation of the franciscana (Pontoporia blainvillei) in the north of Rio de Janeiro, Brazil. Journal of Cetacean Research and Management. v.2, n.2, p.185-192, 2001.

BENEDITTO, A. P. M.; LIMA, N. R. W.. Biometria de teleósteos da costa norte do Estado do Rio de Janeiro para estudos sobre piscivoria. Biotemas, v.16, n.1, p.135-144, 2003.

BENEDITTO, A. P. M.; RAMOS, R. M. A.; LIMA, N. R. W.. Os golfinhos: origem, classificação, captura acidental, hábito alimentar. Porto Alegre: Cinco Continentes, 2001.

BENEDITTO, A. P. M.; RAMOS, R. M. A.; LIMA, N. R. W.. Fishing activity in Northern Rio de Janeiro State (Brazil) and its relation with small cetaceans. Brazilian Archives of Biology and Technology. v.41, n.3, p.296-302, 1998. DOI: http://dx.doi.org/10.1590/S1516-89131998000300004

BENEDITTO, A. P. M.; SOUZA, C. M. M.; KEHRIG, H. A.; REZENDE, C. E.. Use of multiple tools to assess the feeding preference of coastal dolphins. Marine Biology. v.158, n.10, p. 2209-2217, 2011. DOI: http://dx.doi.org/10.1007/s00227$\underline{011-1726-3}$

CAMPOS, L. B.; LOPES, X. M.; SILVA, E.; SANTOS, M. C. O.. Feeding habits of the Franciscana dolphin (Pontoporia blainvillei) in southeastern Brazil. Journal of the Marine Biological Association of the United Kingdom, v.100, n.2, p.301-313, 2020. DOI:

https://doi.org/10.1017/S0025315420000120

DANILEWICZ, D.; ROSAS, F.; BASTIDA, R.; MARIJO, J.; MUELBERT, M.; RODRÍGUEZ, D.; BRITO JUNIOR, J. L.; RUOPOLLO, V.; RAMOS, R.; BASSOI, M.; OTT, P. H.; CAON, G.; ROCHA, A. M.; CATÃO-DIAS, J. L.; SECCHI, E. R.. Report of the Working Group on Biology and Ecology. The Latin American Journal of Aquatic Mammals, v.1, n.1, p.25-42, 2002.

GERACI, J. R.; LOUNSBURY, V. J.. Marine mammals ashore: a field guide for strandings. Galveston: Texas A\&M University, 2005.

OTT, P. H.; SECCHI, E. R.; MORENO, I. B.; DANILEWICZ, D.; CRESPO, E. A.; BORDINO, P.; RAMOS, R.; BENEDITTO, A. P. M.; BERTOZZI, C.; BASTIDA, R.; ZANELATTO, R.; PEREZ, J. E.; KINAS, P. G.. Report of the Working Group on Fishery Interactions. The Latin American Journal of Aquatic Mammals. v.1, n.1, p.55-64, 2002.

SICILIANO, S.; BENEDITTO, A. P. M.; RAMOS, R. M. A.. A toninha, Pontoporia blainvillei (Gervais \& d'Orbigny, 1844) (Mammalia, Cetacea, Pontoporiidae), nos Estados do Rio de Janeiro e Espírito Santo, costa sudeste do Brasil: caracterização dos hábitats e possíveis fatores de isolamento das populações. Boletim do Museu Nacional, Série Zoologia, v.146, n.1, p.1-15, 2002.

ZERBINI, A. N.; SECCHI, E.; CRESPO, E.; DANILEWICZ, D.; REEVES, R.. Pontoporia blainvillei, Franciscana Errata version View on www.iucnredlist.org THE IUCN RED LIST OF THREATENED SPECIES ${ }^{\text {TM }}$. In: DISTRIBUTION AND ABUNDANCE OF FRANCISCANA DOLPHINS IN BRAZIL. Annals. 2019. DOI: https://dx.doi.org/10.2305/IUCN.UK.20173.RLTS.T17978A50371075

A CBPC - Companhia Brasileira de Produção Científica (CNPJ: 11.221.422/0001-03) detém os direitos materiais desta publicação. Os direitos referem-se à publicação do trabalho em qualquer parte do mundo, incluindo os direitos às renovações, expansões e disseminações da contribuição, bem como outros direitos subsidiários. Todos os trabalhos publicados eletronicamente poderão posteriormente ser publicados em coletâneas impressas sob coordenação da Sustenere Publishing, da Companhia Brasileira de Produção Científica e seus parceiros autorizados. Os (as) autores (as) preservam os direitos autorais, mas não têm permissão para a publicação da contribuição em outro meio, impresso ou digital, em português ou em tradução. 